\title{
HIGHLY IONIZED POTASSIUM LINES IN SOLAR X-RAY SPECTRA AND THE ABUNDANCE OF POTASSIUM
}

\author{
J. Sylwester ${ }^{1}$, B. Sylwester ${ }^{1}$, K. J. H. Phillips ${ }^{2}$, And V. D. Kuznetsov ${ }^{3}$ \\ ${ }^{1}$ Space Research Centre, Polish Academy of Sciences, 51-622, Kopernika 11, Wrocław, Poland; js@ @bk.pan.wroc.pl \\ ${ }^{2}$ Mullard Space Science Laboratory, University College London, Holmbury St. Mary, Dorking, Surrey RH5 6NT, UK; kjhp@ mssl.ucl.ac.uk \\ ${ }^{3}$ Institute of Terrestrial Magnetism and Radiowave Propagation (IZMIRAN), Troitsk, Moscow, Russia; kvd@izmiran.ru \\ Received 2009 December 5; accepted 2010 January 6; published 2010 January 21
}

\begin{abstract}
The abundance of potassium is derived from X-ray lines observed during flares by the RESIK instrument on the solar mission CORONAS-F between $3.53 \AA$ and $3.57 \AA$. The lines include those emitted by He-like $\mathrm{K}$ and Li-like K dielectronic satellites, which have been synthesized using the CHIANTI atomic code and newly calculated atomic data. There is good agreement between observed and synthesized spectra, and the theoretical behavior of the spectra with varying temperature estimated from the ratio of the two GOES channels is correctly predicted. The observed fluxes of the He-like $\mathrm{K}$ resonance line per unit emission measure give $\log A(\mathrm{~K})=5.86$ (on a scale $\log A(\mathrm{H})=12$ ), with a total range of a factor 2.9. This is higher than photospheric abundance estimates by a factor 5.5, a slightly greater enhancement than for other elements with first ionization potential (FIP) less than $\sim 10 \mathrm{eV}$. There is, then, the possibility that enrichment of low-FIP elements in coronal plasmas depends weakly on the value of the FIP which for $\mathrm{K}$ is extremely low $(4.34 \mathrm{eV})$. Our work also suggests that fractionation of elements to form the FIP effect occurs in the low chromosphere rather than higher up, as in some models.
\end{abstract}

Key words: line: identification - Sun: abundances - Sun: corona - Sun: flares - Sun: X-rays, gamma rays

Online-only material: color figures

\section{INTRODUCTION}

There continues to be active discussion of why and how solar coronal element abundances differ from those in the photosphere by amounts that depend on the first ionization potential (FIP). It is generally believed that elements with low FIP $(\lesssim 10 \mathrm{eV})$ have coronal abundances that are enhanced over the photospheric abundances (Grevesse et al. 2007) by factors of between $\sim 4$ (Feldman 1992) and $\sim 2$ (Fludra \& Schmelz 1999), while coronal abundances of high-FIP elements are generally within a factor of 2 of photospheric abundances. Models explaining this "FIP effect" rely on some process that separates neutral atoms from ions at the chromospheric level, since there elements with low FIP are at least partly ionized but elements with high FIP are not. Processes such as diffusion along vertical magnetic fields or magnetic fields raising ions into the corona but not neutral atoms (Hénoux \& Somov 1997) have been considered. More recently, Laming $(2004,2009)$ has proposed a model involving ponderomotive forces arising from the generation of Alfvén waves from the chromosphere and into coronal loops. This model has the advantage of explaining the "inverse FIP effect" apparently present in active RS CVn binary systems (Audard et al. 2003), i.e., low-FIP elements having coronal abundances that are depleted compared with photospheric abundances. The model of Hénoux \& Somov (1997) involving electric currents within thin flux tubes involves fractionation at the level of the temperature minimum region and might result in elements with extremely low FIPs to have coronal abundances enhanced by factors larger than 3 or 4. Feldman (1993) has cited evidence that this is the case.

Potassium is of importance in this context as its FIP is the lowest of any common solar element: $4.34 \mathrm{eV}$. There are, however, few potassium spectral lines strong enough to be observable by ultraviolet or X-ray spectrometers. X-ray observations with a spectrometer on the P78-1 spacecraft of the He-like K (K xvIII) line at $3.01 \AA$ (Doschek et al. 1985) give $N(\mathrm{~K}) / N(\mathrm{H})=4.2 \times 10^{-7}$ (or $\log A(\mathrm{~K})=5.62$ on a $\operatorname{logarithmic}$ scale where $\log A(\mathrm{H})=12$ ), based on six summed spectra made up of 72 individual observations. A recent analysis of Solar Ultraviolet Measurement of Emitted Radiation (SUMER) observations by Bryans et al. (2009) uses a K IX line emitted by a quiet coronal region to obtain $\log A(\mathrm{~K})=5.37$. Photospheric determinations give generally lower abundances: Lambert \& Luck (1978) give $\log A(\mathrm{~K})=5.12$, while the more recent work of Takeda et al. (1996), based on a non-local thermodynamic equilibrium analysis of the $\mathrm{K} I$ resonance line at $7699 \AA$, gives $\log A(\mathrm{~K})=5.1$. The comprehensive tabulation of solar abundances by Lodders (2003) includes abundances from CI-type carbonaceous chondrites, for which $\log A(\mathrm{~K})=5.09$, very similar to photospheric values. Comparison of these values suggests that the potassium abundance is enhanced in coronal or flare plasmas over photospheric by a factor between 1.8 and 3.3. This would seem to argue against a FIP mechanism such as those where the fractionation level is at the temperature minimum region, resulting in much larger enhancement factors for elements such as $\mathrm{K}$ with very low FIPs.

The RESIK X-ray spectrometer on the CORONAS-F solar spacecraft obtained numerous spectra in the range 3.4-6.1 $\AA$ in the period from 2001 August to 2003 May. The RESIK instrument (described by Sylwester et al. 2005) was a crystal spectrometer having four channels, the solar X-ray emission being diffracted by silicon and quartz crystals. Pulse-height analyzers enabled solar photons to be distinguished from those produced by fluorescence of the crystal material, enabling the instrumental background to be entirely eliminated for channels 1 and $2(\mathrm{Si}$ 111 crystal, $2 d=6.27 \AA$ ), and its amount accurately estimated for channels 3 and 4 (quartz $10 \overline{1} 0$ crystal, $2 d=8.51 \AA$ ). RESIK was uncollimated to maximize the instrument's sensitivity. Channel 1 of RESIK covers the range 3.40-3.80 $\AA$, and during flares the principal $\mathrm{K}$ XVIII lines with transitions $1 s^{2}-1 s 2 l$ 


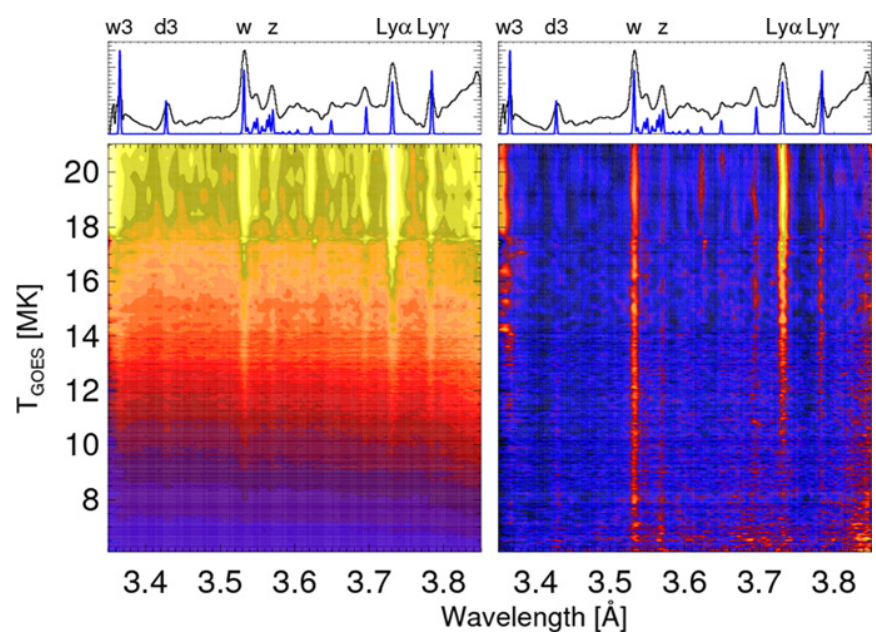

Figure 1. Two thousand seven hundred ninety five RESIK channel 1 spectra stacked with increasing temperature $T_{G O E S}$ (in $\mathrm{MK}$ ) in the vertical direction shown in a gray-scale representation, with lighter areas indicating higher fluxes. The total spectrum is at the top of each plot, together with synthetic spectra (narrow line profiles). Principal line features are shown by letters (see Table 1 for details). Left: not normalized. Right: normalized to the total number of photon counts in each spectrum.

(A color version of this figure is available in the online journal.)

$(l=s, p)$ were observed. This paper reports on an analysis of these spectra, comparing observed and theoretical spectra. Values of the potassium abundance in flare plasmas are derived, which are compared with those expected in theories for the origin of the solar FIP effect.

\section{OBSERVATIONS}

Some 2795 channel 1 spectra during 20 flares were examined, ranging in GOES importance from B9.6 to X1.0, between 2002 August and 2003 February, discussed recently in a separate work (Phillips et al. 2009). Over the period the spectra were taken, the RESIK pulse-height analyzers were close to optimum settings, enabling the solar continuum visible in channel 1 to be accurately estimated. A small level of particle radiation was eliminated using counts in "hidden" (non-solar) bins in the spectra. The times of flares, GOES importance, and heliographic locations were given in a recent study of continuum emission (Phillips et al. 2009) and so will not be repeated here. Individual spectra were collected in data gathering intervals (DGIs) that were inversely related to the flare intensity, with typical DGIs of $2 \mathrm{~s}$ at the peak of strong flares and a few minutes for intervals when the incident X-ray flux was small. Absolute fluxes for the lines and continuum, estimated to have $\sim 20 \%$ accuracy, were derived using the procedure given by Sylwester et al. (2005). A temperature distribution should strictly be used to describe the flare conditions for each spectrum, but we have generally found that an isothermal plasma generally characterizes RESIK spectra adequately in channels 1 and 2. Although there are a number of options for the determination of the single temperature for each spectrum, we elected to use temperatures from the ratio of emission in the two channels of GOES, $T_{G O E S}$ using conversion factors of White et al. (2005); the justification for this is given by Phillips et al. (2006).

Figure 1 shows spectra stacked vertically in a gray-scale representation according to the value of $T_{G O E S}$. The full wavelength scale of channel 1 is shown. Without normalization (left panel), a continuous rise in both the continuum flux and flux in some lines is evident, but when the spectra are normalized by the
Table 1

Principal Lines Observed in RESIK Channel 1

\begin{tabular}{lccc}
\hline \hline \multicolumn{1}{c}{ Ion } & Transition & Wavelength $(\AA)$ & Notation \\
\hline Ar XVII & $1 s^{2}{ }^{1} S_{0}-1 s 3 p^{1} P_{1}$ & 3.366 & $w 3$ \\
Ar XVI & $1 s^{2} n l-1 s 3 p n l$ & 3.428 & $D 3$ \\
K XVIII & $1 s^{2}{ }^{1} S_{0}-1 s 2 p^{1} P_{1}$ & 3.532 & $w$ \\
& $1 s^{2}{ }^{1} S_{0}-1 s 2 p^{3} P_{1}, 1 s 2 p^{3} P_{2}$ & 3.55 & $x+y$ \\
& $1 s^{2}{ }^{1} S_{0}-1 s 2 p^{3} S_{1}$ & 3.571 & $z$ \\
& & & \\
S XVI & $1 s-n p$ & 3.549 & Series limit \\
& & & \\
S XVI & $1 s-7 p$ to $1 s-10 p$ & $3.58-3.62$ & Lyı-Ly $\zeta$ \\
S XVI & $1 s-6 p$ & 3.649 & Ly $\epsilon$ \\
S XVI & $1 s-5 p$ & 3.696 & Ly $\delta$ \\
Ar XVIII & $1 s-2 p$ & 3.731 & Ly $\alpha$ \\
S XVI & $1 s-4 p$ & 3.784 & Ly $\gamma$ \\
\hline
\end{tabular}

total number of photon counts (right panel), the line emission is much clearer. The summed spectra shown at the top of the two plots indicate the principal lines. At the wavelength resolution ( $8 \mathrm{~mA})$ of channel 1 , three KXVIII line features are evident over the entire temperature range (4-22 MK): these are the resonance $(w)$ line, a blend of the intercombination lines $(x+y)$, and the so-called forbidden line $z$, with emission present on the short-wavelength side of $z$ identifiable with K XVII dielectronic satellites. Lines of $\operatorname{Ar}$ XVII $\left(w 3\right.$, transition $1 s^{2}-1 s 3 p$, at $3.666 \AA$ ) and Ar XVIII (Ly $\alpha$ at $3.731 \AA$ ) occur at higher temperatures. This is expected from the contribution functions $G\left(T_{e}\right)$ (line flux per unit emission measure at electron temperature $T_{e}$ ) of these lines, which peak at $T_{e}=24 \mathrm{MK}$ for the K XVIII lines but at $T_{e}>30 \mathrm{MK}$ for the Ar lines. The Lyman series of S XVI lines occurs in this region, with the Ly $\gamma$ line at $3.784 \AA$; higher members of the series are present in spectra of strong flares on the long-wavelength side of the K XVIII lines, though the series limit occurs between the K XVIII $w$ and $z$ lines. Table 1 lists all the observable features that have been identified in RESIK channel 1 spectra with their line identifications including transitions and labels used by various authors.

\section{SYNTHETIC SPECTRA}

To compare RESIK channel 1 spectra, in particular the group of KXVIII lines at $3.53 \AA$ and $3.57 \AA$, with theory, a computer program was written to calculate line fluxes for given electron temperatures $T_{e}$ and emission measures $\left(N_{e}^{2} V\right.$, where $N_{e}=$ electron density and $V=$ the emitting volume) and to synthesize the line and continuum spectrum over the range of channel 1 . The CHIANTI atomic database and code (version 6) include intensity and wavelength data for the K XVIII lines assuming direct collisional excitation from the ground state (based on distorted-wave collision strengths calculated by Zhang \& Sampson (1987)) as well as excitation of K XVIII lines by recombination of $\mathrm{H}$-like $\mathrm{K}$ ions. Ionization fractions needed for this were taken from the recent work of Bryans et al. (2009).

CHIANTI does not include excitation rates for the K XVII dielectronic satellites present near the K XVIII lines, although they make important contributions to the spectra observed by RESIK. $\mathrm{K}$ XVII dielectronic satellites are formed both by dielectronic recombination of the He-like $\mathrm{K}\left(\mathrm{K}^{+17}\right)$ ion and by inner-shell excitation of the Li-like $\mathrm{K}$ ion. The satellites occurring in the region of interest here have transitions like $1 s^{2} n l-1 s 2 p n l$, with $n l$ representing the "spectator" electron. Only satellites in the $1 s^{2} 2 s-1 s 2 s 2 p$ array can be excited by inner-shell excitation for solar plasmas with densities $N_{e} \lesssim 10^{13} \mathrm{~cm}^{-3}$. While 
Table 2

Dielectronic Recombination Excitation of K XVII Lines

\begin{tabular}{lcccc}
\hline \hline \multicolumn{1}{c}{ Transition } & Wavelength $(\AA)$ & $F(s)\left(\mathrm{s}^{-1}\right)$ & Notation $^{\text {a }}$ & Excitation Energy $(\mathrm{keV})^{2}$ \\
\hline $1 s^{2} 4 p^{2} P_{1 / 2}-1 s 2 p 4 p^{2} D_{3 / 2}$ & 3.535 & $4.42(13)$ & 3.246 \\
$1 s^{2} 4 p^{2} P_{3 / 2}-1 s 2 p 4 p^{2} D_{5 / 2}$ & 3.535 & $5.39(13)$ & & 3.246 \\
$1 s^{2} 3 p{ }^{2} P_{1 / 2}-1 s 2 p 3 p{ }^{2} D_{3 / 2}$ & 3.539 & $1.27(14)$ & $d 15$ & 3.049 \\
$1 s^{2} 3 p{ }^{2} P_{3 / 2}-1 s 2 p 3 p{ }^{2} D_{5 / 2}$ & 3.540 & $1.78(14)$ & $d 13$ & 3.049 \\
$1 s^{2} 2 p{ }^{2} P_{3 / 2}-1 s 2 p^{2}{ }^{2} S_{1 / 2}$ & 3.553 & $6.19(13)$ & $m$ & 2.478 \\
$1 s^{2} 2 s{ }^{2} S_{1 / 2}-1 s 2 s 2 p\left({ }^{1} P\right)^{2} P_{3 / 2}$ & 3.559 & $1.23(13)$ & $q$ & 2.440 \\
$1 s^{2} 2{ }^{2} S_{1 / 2}-1 s 2 s 2 p\left({ }^{1} P\right)^{2} P_{1 / 2}$ & 3.562 & $2.86(13)$ & $r$ & 2.440 \\
$1 s^{2} 2 p{ }^{2} P_{3 / 2}-1 s 2 p^{2}{ }^{2} P_{3 / 2}$ & 3.563 & $4.85(13)$ & $a$ & 2.478 \\
$1 s^{2} 2 p{ }^{2} P_{1 / 2}-1 s 2 p^{2}{ }^{2} D_{3 / 2}$ & 3.566 & $2.04(14)$ & $k$ & 2.478 \\
$1 s^{2} 2 p{ }^{2} P_{3 / 2}-1 s 2 p^{2}{ }^{2} D_{5 / 2}$ & 3.569 & $2.79(14)$ & $j$ & 2.478 \\
\hline
\end{tabular}

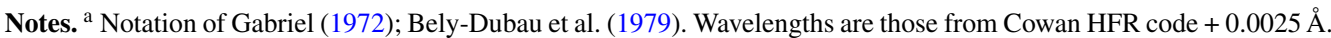

only a small number of the dielectronically formed satellites are significant individually, the cumulative effect of spectator electrons with high $n$ is significant, particularly as their wavelengths converge on the KXVIII $w$ and $y$ lines. We therefore considered a large number of satellites with a wide variety of transition arrays. Wavelengths and intensity factors are needed to synthesize spectra. The flux at the distance of the Earth of a satellite $s$ from a solar plasma with electron temperature $T_{e}$ and volume emission measure $E M=N_{e}^{2} V$ is

$$
\begin{aligned}
I(s)= & 1.66 \times 10^{-16} \frac{N\left(\mathrm{~K}^{+17}\right)}{N(\mathrm{~K})} \frac{N(\mathrm{~K})}{N(\mathrm{H})} \\
& \times \frac{F(s) \exp \left(-\Delta E / k T_{e}\right)}{T_{e}^{3 / 2}} \text { photon } \mathrm{cm}^{-2} \mathrm{~s}^{-1},
\end{aligned}
$$

where the ionization fraction $N\left(\mathrm{~K}^{+17}\right) / N(\mathrm{~K})$ is a function of $T_{e}$ for the flare plasma if close to ionization equilibrium, $N(\mathrm{~K}) / N(\mathrm{H})$ is the abundance of $\mathrm{K}$ relative to $\mathrm{H}, \Delta E$ is the excitation energy of the satellite's upper level with respect to the ground level of the He-like stage, and $F(s)$ is the intensity factor for the satellite, depending on the autoionization and radiative transition rates from the satellite line's upper level (see Phillips et al. 1994 for more details). To calculate the energy levels and the $F(s)$ factors, we ran the HartreeFock atomic code of Cowan (1981) with pseudorelativistic corrections (HFR). The code in earlier forms could only be run on supercomputers, but adaptations have been made (A. Kramida 2008, private communication) such that the code now runs on modest sized personal computers, which was how the code was run for this case. Some 418 transitions with spectator electrons $n l$ up to $6 d$ were considered. Input to the HFR code for the calculation of satellite line data includes scaling factors for Slater parameters which slightly affect the energy levels and therefore the line wavelengths. Our previous calculations (Phillips et al. 1994) have used $100 \%$ scaling which produces line wavelengths only a few $m \AA$ from observed values in the case of Ar and Fe. Unfortunately, there are no accurate observed wavelengths of K XVII satellites available. Use of $100 \%$ scaling for the Slater parameters in the case of K XVII satellites produced wavelengths for high- $n$ satellites converging on $3.529 \AA$ and $3.544 \AA$, about $0.0025 \AA$ shorter than the wavelengths of the KXVIII $w$ and $y$ lines. The wavelengths of all satellites were therefore adjusted in the synthesis computer program by adding $0.0025 \AA$ to the wavelengths from the Cowan program. This wavelength adjustment results in KXVII satellite $j$ blending with K XVIII line $z$ if the line widths are due solely to thermal Doppler broadening. This blending of $j$ and $z$ in $\mathrm{Ca}$ spectra was observed with the Bent Crystal Spectrometer (BCS) on Solar Maximum Mission (SMM; Bely-Dubau et al. (1982): note $Z(\mathrm{Ca})=20, Z(\mathrm{~K})=19)$, and gives further support for the way in which we adjusted the satellite wavelengths. The most important dielectronically formed satellites are listed, with their transitions, adjusted wavelengths, and $F(s)$ values, in Table $2 .^{4}$

The satellites in the K XVII $1 s^{2} 2 s-1 s 2 s 2 p$ array are also excited by inner-shell excitation of the Li-like ion, with lines $q$ and $r$ making significant contributions to the spectrum. Excitation data for these satellites were calculated using collision strengths given by Bely-Dubau et al. (1982) for the case of the equivalent Ca XVIII satellites, and ion fractions from Bryans et al. (2009).

CHIANTI does not give data for the higher- $n$ members of the S XVI Lyman series, some of which are important for a complete synthesis of the spectral region. The generally weak S XVI lines that are close to the K XVIII lines were included by a straight extrapolation of fluxes available from CHIANTI for lines with transitions $1 s-n p, n \leqslant 5$ (data from interpolation of $R$-matrix calculations by Aggarwal \& Kingston 1991). This is justified by the fact that de-excitation of the $n p$ levels is almost entirely by transitions to the ground state $1 s^{2} S_{1 / 2}$ and not to intermediate states. The wavelengths of these lines were from Kelly (1987) and Erickson (1977). Data for the Ar XVII and Ar XVIII lines were taken from CHIANTI.

The continua in the range of RESIK channel 1 are principally due to free-free and free-bound emission. They were derived from CHIANTI routines as discussed more fully by Phillips et al. (2009). Free-free emission is almost entirely due to $\mathrm{H}$ and $\mathrm{He}$, but free-bound emission is due to recombination on to the ions of a variety of elements, in particular to (in descending order of importance) $\mathrm{Si}, \mathrm{Fe}, \mathrm{Mg}$, and $\mathrm{O}$. A choice of element abundances must therefore be made, and for our purposes we chose a coronal abundance set given in the CHIANTI atomic database (sun_corona_ext.abund), based on Feldman (1992). The ion fractions were taken from Bryans et al. (2009). Recombination on to fully stripped $\mathrm{S}$ gives rise to a small recombination edge at $3.548 \AA$, between the K XVIII $x$ and $y$ lines, but our synthesis program indicates that it does not make a significant contribution to the spectrum.

Finally, in the calculation of synthetic spectra, values of the spectral line widths must be specified. For an instrument with perfect spectral resolution, these would normally be determined by thermal Doppler broadening, with temperatures in the observed range 4-22 MK of the spectra analyzed. There is generally an additional broadening due to nonthermal motions of the emitting ions, with typical velocities of several tens of $\mathrm{km} \mathrm{s}^{-1}$ for

\footnotetext{
4 Full results of the calculations are available from the authors.
} 

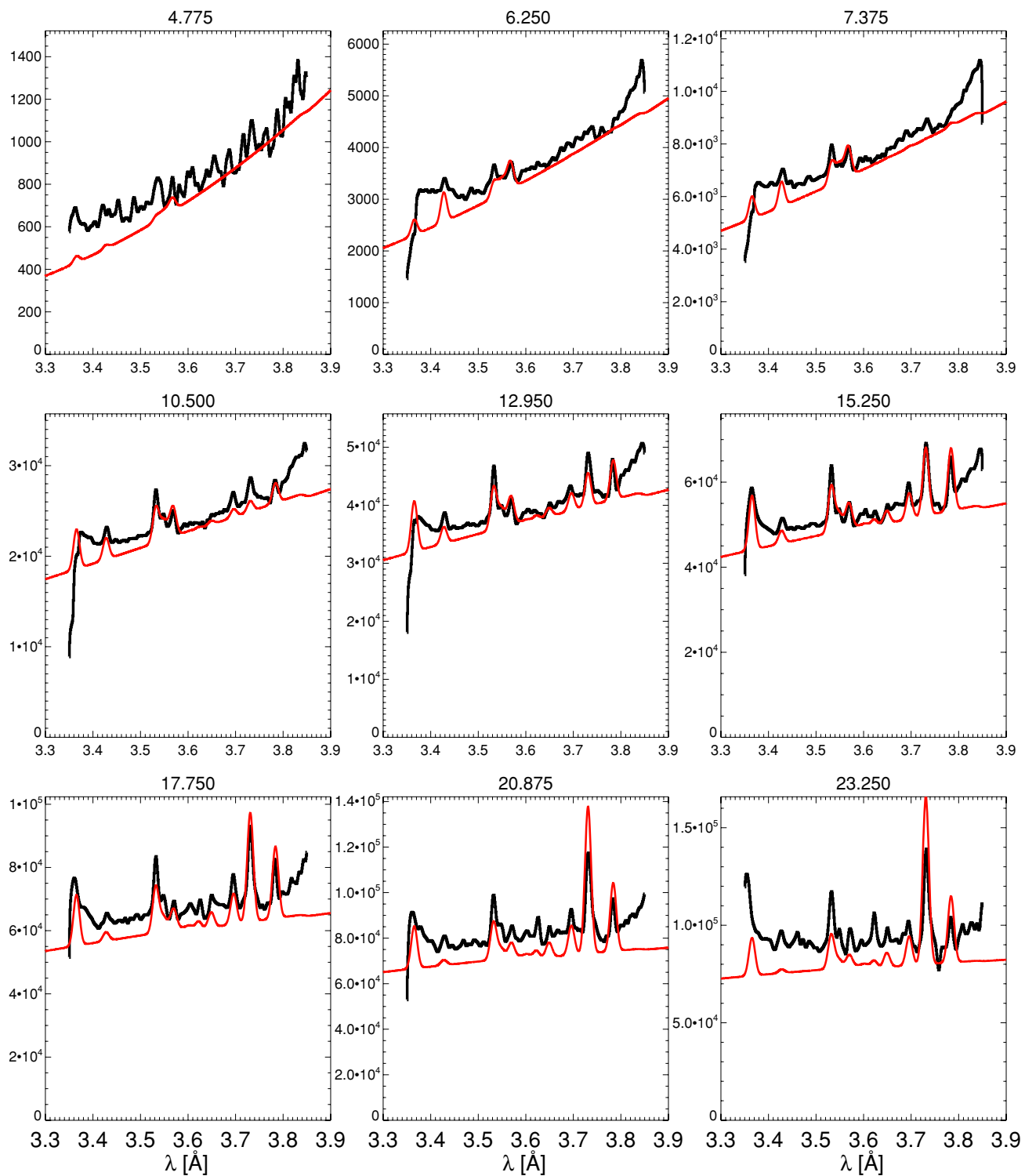

Figure 2. RESIK channel 1 spectra compared with synthetic spectra for nine temperatures (values indicated above each plot in MK), with spectral irradiance (photons $\mathrm{cm}^{-2} \mathrm{~s}^{-1} \AA^{-1}$ ) along the vertical axes. The $\mathrm{K}$ XVIII lines are between $3.532 \AA$ and $3.571 \AA$ (other line features are identified in Table 1 ).

(A color version of this figure is available in the online journal.)

flare spectra. The combined widths amount to $2 \mathrm{~m} \AA$ (FWHM) at most. In evaluating synthetic spectra, thermal Doppler broadening only was taken into account, the line widths being dominated by the instrumental width which for RESIK channel 1 is $8 \mathrm{~m} \AA$ (FWHM).

\section{RESULTS}

\subsection{Comparison of Observed and Synthetic Spectra}

The observed RESIK spectra were compared with those synthesized as described in Section 3 with temperature and emission measure from the ratio of the two GOES channels. Figure 2 shows a selection of nine RESIK spectra from a single flare with $T_{G O E S}$ over the interval 4-22 MK. For the low-temperature spectra $\left(T_{G O E S} \lesssim 10 \mathrm{MK}\right)$, there is a steep rise of the observed continuum emission with wavelength, very similar to the rise in theoretical free-bound and freefree emission. At these low temperatures, the slope of the theoretical continuum is extremely sensitive to temperature.
Only at temperatures $\gtrsim 6 \mathrm{MK}$ is any line emission evident in the RESIK spectra. For $T_{G O E S} \lesssim 10 \mathrm{MK}$, the principal lines are the K XVIII lines and the Ar XVI D3 satellite feature made up of transitions $1 s^{2} n l-1 s 3 p n l$ at $3.428 \AA$. The feature at the end of the range, at $\sim 3.84 \AA$, is unidentified and may not be solar in origin; it may for example be due to a curvature effect in the diffracting crystal. At higher temperatures, the $\mathrm{K}$ XVIII line triplet, made up of lines $w, x+y$, and $z$ with blended $\mathrm{K}$ XVII satellites, becomes prominent, as do the $\mathrm{S}$ XVI Lyman lines and ArXVII and Ar XVIII lines included in Table 1. The observed continuum becomes larger than that in the synthetic spectra, up to $15 \%$ by $T_{G O E S}=22 \mathrm{MK}$, which may be due to an increasing departure from an isothermal plasma. For the K XVIII lines, the $w$ line becomes steadily stronger compared with the blend of the $z$ line with the $\mathrm{KXVII} j$ and $k$ satellites, as predicted by the synthetic spectra, the flux of the dielectronic satellites having an approximately $T_{e}^{-1}$ dependence with respect to line $w$ (Gabriel 1972).

In summary, the comparison of observed and synthetic spectra shows fair agreement, especially for the KXVIII lines and 

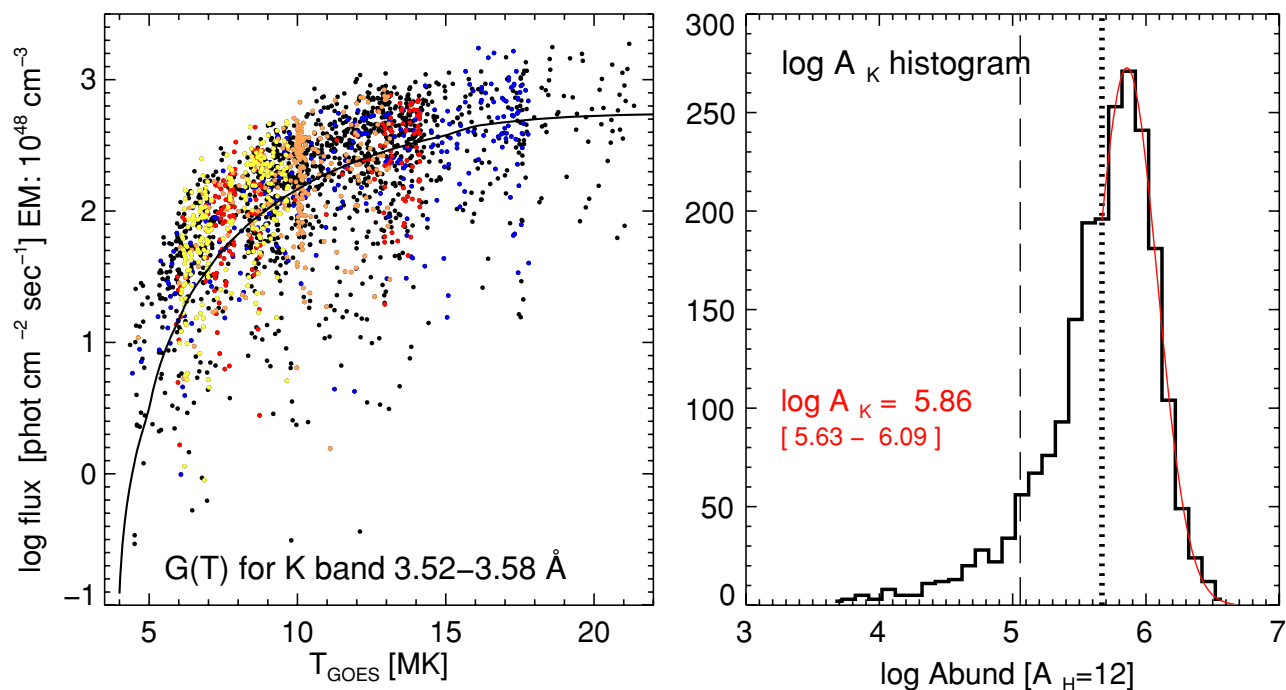

Figure 3. Left: flux of K XVIII $w$ line (including unresolved dielectronic satellites) normalized to an emission measure of $10^{48} \mathrm{~cm}^{-3}$ (derived from GOES) plotted against $T_{G O E S}$. Right: number distribution of estimates of the deduced $\mathrm{K}$ abundance. The peak of the distribution corresponds to a $\mathrm{K}$ abundance of $\log A(\mathrm{~K})=5.86$, with half-width corresponding to the range 5.63-6.09. The previous coronal (flare) abundance estimate of Doschek et al. (1985) is shown with the dotted vertical line, the photospheric estimates of Lambert \& Luck (1978) and Takeda et al. (1996) by the dashed vertical line.

(A color version of this figure is available in the online journal.)

nearby K XVII satellites, which confirms our earlier results for continuum emission (Phillips et al. 2009) that a single value of temperature is valid for this narrow spectral range except for high temperatures $\left(T_{G O E S} \gtrsim 20 \mathrm{MK}\right)$.

\subsection{Abundance of Potassium}

The RESIK observations clearly show the KXVIII lines over a wide temperature range. Using line-fitting routines, we estimated the fluxes of the line features $w, x+y$, and $z$ and, with temperature and emission measure derived from GOES, we derived the absolute abundance of $\mathrm{K}$. The line flux for an emission measure of $10^{48} \mathrm{~cm}^{-3}$ is plotted for each RESIK spectrum against $T_{G O E S}$ in Figure 3 (left panel). The scatter in the 2795 points indicates the measurement uncertainties which are, as expected, greater for weaker, low-temperature spectra. A few outliers have small line fluxes for rather high temperatures which are mostly at the initial times of flares when the isothermal assumption with temperature given by $T_{G O E S}$ is least accurate. Any flare-to-flare variations, as was found from SMM data (Sylwester et al. 1984), appear to be less than the scatter of the points. The theoretical curve shown is the contribution function $G\left(T_{e}\right)$ for the sum of the $\mathrm{K}$ XVIII lines and blended KXVII satellites for an emission measure $10^{48} \mathrm{~cm}^{-3}$. The abundance of $\mathrm{K}$ assumed in this curve is $\log A(\mathrm{~K})=5.86$.

Figure 3 (right panel) shows the individual determinations of the absolute abundance of $\mathrm{K}$ from these spectra plotted as a histogram, with the logarithm of the abundance as the abscissa. These determinations were made from the departure of individual points from the theoretical curve shown in the figure. The peak of this distribution corresponds to an abundance $\log A(\mathrm{~K})$ of 5.86, with the half-width of the distribution corresponding to the range $\log A(\mathrm{~K})=5.63-6.09$, i.e., a factor of 2.9. This value updates the determination of Phillips et al. (2003) $(\log A(\mathrm{~K})=$ 5.57) based on RESIK spectra during long-duration flares with a preliminary calibration and for periods when the pulse-height analyzers were not quite at optimum operation.

This may be compared with the $\mathrm{K}$ abundance estimates given earlier. Our value is higher than that of Doschek et al. (1985) by a factor 1.7, though our uncertainty range only just excludes it. It is higher by a factor of 3 than that of Bryans et al. (2009) from SUMER measurements a quiet solar region, and is a factor 5.5 higher than the photospheric determinations of Lambert \& Luck (1978) and Takeda et al. (1996), and meteoritic value given by Lodders (2003). The factor of 2 range of our values from 20 flares covers a wide range of flares, though any flare-toflare variations (Sylwester et al. 1984) are apparently less than the scatter of the points. Our results suggest that the coronal abundance enhancements of very low FIP elements may increase slightly with decreasing FIP, as is suggested by the FIP model of Hénoux \& Somov (1997), though the amount is too small to make a definitive comment.

This abundance determination of $\mathrm{K}$ and previous work relating to other low-FIP elements raise the possibility of tracing the origin of flare plasma. Assuming that this plasma originates by chromospheric evaporation at the flare onset, a chromospheric origin of the plasma is expected with abundances that reflect this origin. The models proposed by Laming (2004) and Laming (2009) for the FIP effect were based on a fractionation level in the high chromosphere, so in the flare evaporation phase plasma with essentially photospheric origin (i.e., below the fractionation level) is delivered into the flare loops. However, there are some lines of evidence suggesting that the fractionation may occur in the lower chromosphere, below the level at which chromospheric evaporation occurs, so that coronal abundances are generally observed in flare plasmas. The present work on the abundance of $\mathrm{K}$ in flares appears to support the view that fractionation of elements occurs low in the chromosphere.

\section{CONCLUSIONS}

The RESIK spectrometer has observed the He-like $\mathrm{K}$ ion $\mathrm{X}$-ray lines in numerous flare spectra, and synthetic spectra based partly on the CHIANTI atomic database and partly on new calculations presented here of K XVII dielectronic satellites compare well with the observations for temperatures and emission measures taken from the ratio of the two GOES channels. The increase of the K XVIII line $w$ relative to the nearby $z$ line at 
higher temperatures, due to the decreasing contribution of satellites, is evident in RESIK spectra. Flux measurements of this line are used to derive the abundance of $\mathrm{K}$ for these flare plasmas with temperatures and emission measures from GOES. Comparison with theory gives a distribution of possible values of the abundance of $\mathrm{K}$ with peak at $\log A(\mathrm{~K})=5.86$ and half-peak range 5.63-6.09. The value 5.86 is higher than the value from X-ray flares of Doschek et al. (1985) (by a factor 1.7) and the value from quiet-Sun SUMER spectra of Bryans et al. (2009) (by a factor 3 ). It is higher by a factor 5.5 than photospheric and meteoritic abundances of $\mathrm{K}$. This indicates that the abundance of $\mathrm{K}$ in flare plasmas obeys the FIP effect as discussed by Feldman (1992) and others, though there is a suggestion that the enhancement of $\mathrm{K}$, the FIP of which is only $4.34 \mathrm{eV}$, is slightly more than low-FIP elements such as $\mathrm{Mg}$, $\mathrm{Si}$, and $\mathrm{Fe}$ with higher FIP. The factor of 2 range of our estimates appears to be uncertainties rather than flare-to-flare variations, as has been observed for the case of Ca (Sylwester et al. 1984). In addition, our work supports the view that the fractionation of elements giving rise to the FIP effect occurs in the low chromosphere, not the high chromosphere, as has been commonly proposed in the past.

We are grateful for financial help from the European Commission's Seventh Framework Programme (FP7/20072013) under grant agreement no. 218816 (SOTERIA project, www.soteria-space.eu), the Polish Ministry of Education and Science grant no. N203 381736, and to the UK-Royal Society/ Polish Academy of Sciences International Joint Project (grant number 2006/R3) for travel support. CHIANTI is a collaborative project involving Naval Research Laboratory (USA), the Universities of Florence (Italy) and Cambridge (UK), and George Mason University (USA). Dr Alexander Kramida at the National Institute of Standards and Technology is thanked for his considerable help in running the Cowan HFR code on small computers.

More information is available at http://www.aas.org/public ations/aastex.

\section{REFERENCES}

Aggarwal, K. M., \& Kingston, A. E. 1991, J. Phys. B, 24, 4583

Audard, M., Güdel, M., Sres, A., Raassen, A. J. J., \& Mewe, R. 2003, A\&A, 398, 1137

Bely-Dubau, F., Gabriel, A. H., \& Volonté, S. 1979, MNRAS, 186, 405

Bely-Dubau, F., et al. 1982, MNRAS, 201, 1155

Bryans, P., Landi, E., \& Savin, D. W. 2009, ApJ, 691, 1540

Cowan, R. D. 1981, The Theory of Atomc Structure and Spectra (Berkeley, CA: Univ. California Press)

Doschek, G. A., Feldman, U., \& Seely, J. F. 1985, MNRAS, 217, 317

Erickson, G. W. 1977, J. Phys. Chem. Ref. Data, 6, 831

Feldman, U. 1992, Phys. Scr., 46, 202

Feldman, U. 1993, ApJ, 411, 896

Fludra, A., \& Schmelz, J. T. 1999, A\&A, 348, 286

Gabriel, A. H. 1972, MNRAS, 160, 99

Grevesse, N., Asplund, M., \& Sauval, A. J. 2007, Space Sci. Rev., 130, 105

Hénoux, J. C., \& Somov, B. V. 1997, A\&A, 318, 947

Kelly, R. L. 1987, Atomic and Ionic Spectrum Lines Below 2000 Angstroms (Melville, NY: AIP)

Lambert, D. L., \& Luck, R. E. 1978, MNRAS, 183, 79

Laming, J. M. 2004, ApJ, 614, 1063

Laming, J. M. 2009, ApJ, 695, 954

Lodders, K. 2003, ApJ, 591, 1220

Phillips, K. J. H., Dubau, J., Sylwester, J., \& Sylwester, B. 2006, ApJ, 638, 1154

Phillips, K. J. H., Keenan, F. P., Harra, L. K., McCann, S. M., Rachlew-Källne, E., Rice, J. E., \& Wilson, M. 1994, J. Phys. B, 27, 1939

Phillips, K. J. H., Sylwester, J., Sylwester, B., \& Kuznetsov, V. D. 2009, ApJ, (submitted)

Phillips, K. J. H., Sylwester, J., Sylwester, B., \& Landi, E. 2003, ApJ, 589, L113

Sylwester, J., Lemen, J. R., \& Mewe, R. 1984, Nature, 310, 665

Sylwester, J., et al. 2005, Sol. Phys., 226, 45

Takeda, Y., Kato, K., Watanabe, Y., \& Sadakane, K. 1996, PASJ, 48, 511

White, S. M., Thomas, R. J., \& Schwartz, R. A. 2005, Sol. Phys., 227, 231

Zhang, H., \& Sampson, D. H. 1987, ApJS, 63, 487 